\title{
Tripping out on TRPV4
}

\author{
Friedrich C. Luft $^{1}$
}

Published online: 24 October 2015

(C) Springer-Verlag Berlin Heidelberg 2015

Transient receptor potential (TRP) ion channels are located on the plasma membrane of most animal cells. TRP channels were initially discovered in the trp-mutant strain of the fruit fly, Drosophila melanogaster. Subsequently, TRP channels were found in vertebrates where they are ubiquitously expressed in many cells and tissues. Almost 30 TRP channels have been described that share some structural similarity with each other and are subdivided into seven subfamilies by sequence similarity: TRPC for canonical, TRPV for vanilloid, TRPM for melastatin, TRPN (named for the first member of the Drosophila NOMPC protein/"no mechanoreceptor potentials") and TRPA (named for its ankyrin repeat domain), TRPP for polycystic, and TRPML for mucolipin. Many of these channels mediate sensations such as pain, temperature, different kinds of tastes, pressure, and vision. Molecules found in spices such as garlic (allicin), chili pepper (capsaicin), and wasabi (allyl isothiocyanate) activate some TRP channels. Others are activated by menthol, camphor, peppermint, and cooling agents. Molecules found in cannabis still activate others. Some TRP channels act as sensors of osmotic pressure, volume, stretch, and vibration. TRP channels are relatively nonselectively permeable to cations, including sodium, calcium, and magnesium. Most TRP channels are composed of six membrane-spanning helices with intracellular Nand C-termini (Fig. 1).

TRPV4, initially named vanilloid receptor-related osmotically activated channel (VR-OAC), and OSM9-like transient

Friedrich C. Luft

luft@charite.de

1 Experimental and Clinical Research Center, Charité Medical Faculty and the Max Delbrück Center for Molecular Medicine,

Lindenbergerweg 80, Berlin 13125, Germany receptor potential channel, member 4 (OTRPC4), is a $\mathrm{Ca}^{2+}$ permeable, nonselective cation channel that has been found involved in multiple physiologic functions, dysfunctions, and also diseases. It functions in the regulation of systemic osmotic pressure by the brain, in vascular function, in liver, intestinal, renal and bladder function, in skin barrier function and response of the skin to ultraviolet-B radiation, in growth and structural integrity of the skeleton, in function of joints, in airway and lung function, in retinal and inner ear function, and in pain. The channel is activated by osmotic, mechanical, and chemical cues. It also responds to thermal changes (warmth). Channel activation can be sensitized by inflammation and injury. Hereditary channelopathy mutations of TRPV4 lead to skeletal dysplasias, premature osteoarthritis, and neurological motor function disorders as a manifestation of a motor neuropathy or spinal muscular atrophy.

My associates identified afferent neurons in the thoracic dorsal root ganglia of mice that innervate hepatic blood vessels and detect physiological hypo-osmotic shifts in blood osmolality [1]. I was able to play a modest role in this project. We found that hepatic sensory neurons are equipped with an inward current that faithfully transduces graded changes in osmolality within the physiological range ( 15 mOsm). In mice lacking the osmotically activated ion channel, TRPV4, hepatic sensory neurons no longer exhibited osmosensitive inward currents and activation of peripheral osmoreceptors in vivo was abolished. We thus identified a new population of sensory neurons that transduce ongoing changes in hepatic osmolality. The findings explained clinical observations made by our group showing that just by drinking cool tap water, blood pressure and basal metabolic rate increased perceptibly $[2,3]$. Since then, TRPV4 has become an "old friend".

In this month's J Mol Med, Fichna et al. identify a new role for TRPV4 in controlling gastrointestinal motility [4]. TRPV4 was implicated in mediating abdominal pain and signs of 


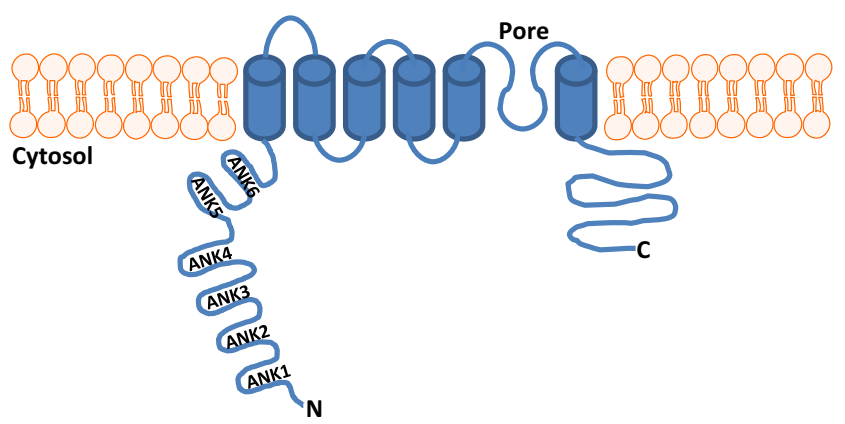

Fig. 1 The TRPV4 protein consists of a cytosolic N-terminal region and six transmembrane domains, including the pore region and an intracellular $\mathrm{C}$-terminal tail. The $\mathrm{N}$-terminal region contains the ankyrin repeat domain (ARD), which consists of six ankyrin repeats (ANK)

intestinal inflammation. The investigators first determined gastrointestinal TRPV4 expression at mRNA and protein levels and then inspected gut motility assays. They then used $\mathrm{Ca}^{2+}$ and nitric oxide (NO) imaging to study intracellular signaling. They "translated" their findings to human colonic epithelium. They basically found that pharmacological TRPV4 activation decreased gastrointestinal motility and increased inhibitory neurotransmission. The investigators demonstrated that TRPV4 reduced NO-dependent $\mathrm{Ca}^{2+}$ release from enteric neurons. They conclude that TRPV4 is involved in regulating the gastrointestinal tract's motility. Since the motility is obviously disturbed in numerous diseases, the clinical implications are obvious.

The enteric nervous system exerts local control over mixing and propulsive movements in the small intestine [5]. When digestion is in progress, intrinsic primary afferent neurons are activated by the contents of the intestine. These have been physiologically characterized in the intrinsic myenteric ganglia. The circular muscle contracts as an annulus, about $2-$ $3 \mathrm{~mm}$ in minimum oral-to-anal extent in the small intestine. The smooth muscle cells form an electrical syncytium that is innervated by about 300 excitatory and 400 inhibitory motor neurons per millimeter length. The intrinsic nerve circuits that control mixing and propulsion in the small intestine are now known. However, how they are programmed to generate the motility patterns that are observed remains to be determined. The knowledge that TRPV4 plays a role is a definite advance.

Astonishing is the fundamental importance of TRPV4 in the entire body. The current evidence about signaling determinants of TRPV4 activation by luminal flow in the distal nephron and how dysfunction of this mechanism contributes to the progression of polycystic kidney disease has recently been reviewed [6]. The known roles for TRPV4 in pulmonary diseases, including pulmonary edema formation, pulmonary hypertension, and acute lung injury have been discussed as have promising aspects and potential pitfalls of the clinical use of TRPV4 inhibitors [7]. We certainly do not want to leave the cardiologists out of the act. The potential role of TRPV4 channels in different forms of ischemic conditioning (pre/post/ remote)-induced cardioprotection has been described using knockout mice and various pharmacological modulators. The role of TRPV4 channels in mediating preconditioninginduced preservation of vascular function in terms restoring NO- and further improving endothelial-mediated endothelial relaxation has been described [8]. The gastroenterologists are by no means left out. TRPV4 enables the digestive system to survey its physical and chemical environment, which is relevant to all processes of digestion [9].

The associated TRPV4 disorders have been categorized and are currently grouped into neuromuscular disorders and skeletal dysplasias; however, the overlap within and between both groups is considerable. Bilateral progressive sensorineural hearing loss occurs in both. Charcot-Marie-Tooth disease type $2 \mathrm{C}$, scapuloperoneal spinal muscular atrophy, and congenital distal spinal muscular atrophy have been attributed to TRPV4. Six skeletal dysplasias, namely familial digital arthropathy-brachydactyly, autosomal dominant brachyolmia, spondylometaphyseal dysplasia, spondyloepiphyseal dysplasia, parastremmatic dysplasia, and metatropic dysplasia have all implicated TRPV4 variants [10]. Can one gene and one ion channel be responsible for so much human havoc?

Perhaps we should look more closely at the channel [11]. How can one channel cause an inherited arthropathy of hands and feet [12] and also mediate osmoreceptor activities in the brain and liver [1]? We have excellent data on the structure of TRPV4 [13]. Looks like a fairly generic TRP channel. And perhaps, that is just the conclusion. How can one channel class cause so many diverse diseases? You did not expect it to be simple, did you?

Respectfully,

Fred Luft

\section{References}

1. Lechner SG, Markworth S, Poole K, Smith ES, Lapatsina L, Frahm S, May M, Pischke S, Suzuki M, Ibanez-Tallon I et al (2011) The molecular and cellular identity of peripheral osmoreceptors. Neuron 69:332-344

2. Boschmann M, Steiniger J, Franke G, Birkenfeld AL, Luft FC, Jordan J (2007) Water drinking induces thermogenesis through osmosensitive mechanisms. J Clin Endocrinol Metab 92: 3334-3337

3. Lipp A, Tank J, Franke G, Arnold G, Luft FC, Jordan J (2005) Osmosensitive mechanisms contribute to the water drinkinginduced pressor response in humans. Neurology 65:905-907

4. Fichna J, Poole DP, Veldhuis N, MacEachern SJ, Saur D, Zakrzewski PK, Cygankiewicz AI, Mokrowiecka A, MaleckaPanas E, Krajewska WM et al (2015) Transient receptor potential vanilloid 4 inhibits mouse colonic motility by activating NOdependent enteric neurotransmission. J Mol Med (Berl). doi:10. 1007/s00109-015-1336-5

5. Kunze WA, Furness JB (1999) The enteric nervous system and regulation of intestinal motility. Annu Rev Physiol 61:117-142 
6. Mamenko M, Zaika O, Boukelmoune N, O'Neil RG, Pochynyuk O (2015) Deciphering physiological role of the mechanosensitive TRPV4 channel in the distal nephron. Am J Physiol Renal Physiol 308:F275-286

7. Goldenberg NM, Ravindran K, Kuebler WM (2015) TRPV4: physiological role and therapeutic potential in respiratory diseases. Naunyn Schmiedeberg's Arch Pharmacol 388:421-436

8. Randhawa PK, Jaggi AS (2015) TRPV1 and TRPV4 channels: potential therapeutic targets for ischemic conditioning-induced cardioprotection. Eur J Pharmacol 746:180-185

9. Holzer P (2011) Transient receptor potential (TRP) channels as drug targets for diseases of the digestive system. Pharmacol Ther 131:142-170

10. Schindler A, Sumner C, Hoover-Fong JE (1993) TRPV4associated disorders. In: Pagon RA, Adam MP, Ardinger HH,
Wallace SE, Amemiya A, Bean LJH, Bird TD, Dolan CR, Fong CT, Smith RJH, Stephens K (eds) GeneReviews (R), Seattle (WA)

11. Garcia-Elias A, Mrkonjic S, Jung C, Pardo-Pastor C, Vicente R, Valverde MA (2014) The TRPV4 channel. Handb Exp Pharmacol 222:293-319

12. Lamande SR, Yuan Y, Gresshoff IL, Rowley L, Belluoccio D, Kaluarachchi K, Little CB, Botzenhart E, Zerres K, Amor DJ et al (2011) Mutations in TRPV4 cause an inherited arthropathy of hands and feet. Nat Genet 43:1142-1146

13. Auer-Grumbach M, Olschewski A, Papic L, Kremer H, McEntagart ME, Uhrig S, Fischer C, Frohlich E, Balint Z, Tang B et al (2010) Alterations in the ankyrin domain of TRPV4 cause congenital distal SMA, scapuloperoneal SMA and HMSN2C. Nat Genet 42: $160-164$ 